\title{
Expression of the copper transporters hCtr1, ATP7A and ATP7B is associated with the response to chemotherapy and survival time in patients with resected non-small cell lung cancer
}

\author{
TIAN YANG, MINGWEI CHEN, TIANJUN CHEN and ASMITANANDA THAKUR \\ Department of Respiratory and Critical Care Medicine, \\ The First Affiliated Hospital of Medical College of Xi'an Jiaotong University, Xi'an, Shaanxi 710061, P.R. China
}

Received September 4, 2014; Accepted June 5, 2015

DOI: $10.3892 / 01.2015 .3531$

\begin{abstract}
Copper transporter family proteins may regulate the chemoresistance of non-small cell lung cancer (NSCLC) to platinum-based anticancer drugs. The present study aimed to investigate the expression of these proteins in lung cancer tissue specimens for association with clinicopathological data and patient responses to chemotherapy and survival. A total of 54 patients with surgically resected NSCLC that received first-line platinum-based doublet chemotherapy were recruited in the present study, and the paraffin-embedded pre-treatment tumor tissue specimens were subjected to immunohistochemical analysis for the expression of human copper transporter 1 (hCtrl) and copper-transporting p-type adenosine triphosphatase 1 (ATP7A) and 2 (ATP7B). This cohort of patients with NSCLC received platinum-based chemotherapy subsequent to the surgical removal of tumor lesions. ATP7B expression was found to be significantly associated with tumor cell differentiation, while hCtrl expression was significantly associated with improved chemotherapeutic responses. The median survival time was 20 months in patients possessing tumors with high ATP7A expression, but $>66$ months in patients possessing tumors with low ATP7A expression at the end of the follow-up $(\mathrm{P}<0.001)$. The median survival time at the end of the follow-up was 15 months in patients with low tumor hCtr1 expression, but $>66$ months in patients with high tumor hCtr1 expression $(\mathrm{P}<0.001)$. High hCtr1 and low ATP7A expression were each favorable prognostic factors subsequent to chemotherapy for patients with resected NSCLC. Multivariate analysis revealed that high hCtrl expression combined with low ATP7A expression, good tumor differentiation and female gender were all favorable independent predictive and
\end{abstract}

Correspondence to: Professor Mingwei Chen, Department of Respiratory and Critical Care Medicine, The First Affiliated Hospital of Medical College of Xi'an Jiaotong Unversity, 277 West Yanta Road, Xi'an, Shaanxi 710061, P.R. China E-mail: mingweichencn@126.com

Key words: copper transporters, NSCLC, platinum prognostic factors for patients with resected NSCLC following chemotherapy. High hCtrl expression combined with low ATP7A expression was associated with an improved prognosis in patients with resected NSCLC that received platinum-based chemotherapy. Surgery combined with neoadjuvant chemotherapy may improve the survival time of patients with NSCLC.

\section{Introduction}

Lung cancer is the most notable health problem among men and women worldwide, accounting for an estimated 1.6 million novel cases per year and $\sim 13 \%$ of total cancer cases diagnosed in 2008 (1,2). At present, lung cancer remains the leading cause of cancer-associated mortality in men and the second leading cause of cancer-associated mortality in women (1). Although lung cancer consists of several histological types, almost $80 \%$ of patients are diagnosed with non-small cell lung cancer (NSCLC). The current treatment options for NSCLC are determined by the stage of the disease and include surgery, chemotherapy and targeted therapy. However, the majority of patients with NSCLC are diagnosed at the advanced stage of disease, and curative surgery is not possible for these patients. In addition, survival with early stage NSCLC is also improved by chemotherapy following surgery (3). Chemotherapy may be administered alone or in combination with surgery or radiation therapy, and platinum-based anti-tumor agents, particularly cisplatin, have been widely used for the treatment of various human cancers, including NSCLC $(4,5)$. These agents are initially effective in lung cancer patients, but due to the development of resistance and relapse in treated patients, the benefit of these platinum based compounds has been limited. Thus, the overall response rate is only $25-30 \%$, and the median survival time is 9-11 months for patients with NSCLC (6-8).

Molecularly, platinum-resistance is multifaceted (9-11). One mechanism of resistance is the reduction in the intracellular accumulation of the drug, which occurs due to the impairment of drug intake, enhancement of outward transport or a combination of the two in tumor cells (1). Thus, additional understanding of the underlying mechanism of platinum resistance may aid in the improvement of the effectiveness of platinum therapy and the response to platinum agents. Previous 
studies have revealed that copper ions and platinum drugs may share the same transport system in the cell (12-14). A different study demonstrated that copper ion transport proteins not only participate in the metabolism of the copper ions, but also maintain the balance of copper and are ultimately associated with the development of cisplatin resistance (15). In particular, human copper transporter 1 (hCtr1) is the major copper influx transporter and also transports cisplatin and cisplatin analogues into cells (13), while two other copper transporters, consisting of copper-transporting p-type adenosine triphosphatase 1 (ATP7A) and 2 (ATP7B), regulate the efflux of cisplatin. A previous study has reported that hCtrl expression is able to predict the prognosis of patients with advanced NSCLC that received platinum-based chemotherapy (16). Thus, in the present study, the expression of hCtr1, ATP7A and ATP7B proteins was detected in surgically resected NSCLC tissues to determine the association between the expression of these proteins with the histology, chemotherapy response, tumor stage, differentiation and prognosis in patients with resected NSCLC that received platinum-based treatment.

\section{Patients and methods}

Patients. In total, 54 patients with a histologically confirmed diagnosis of NSCLC were diagnosed at The First Affiliated Hospital of Xi'an Jiaotong University (Xi'an, Shaanxi, China) between 2005 and 2009, and these patients were enrolled in the present study. These patients underwent surgical resection of the tumor lesions. The patients were enrolled in the present study according to certain criteria. Firstly, surgical resection was required as the first-line treatment, followed by at least two courses of platinum based doublet, but not single or triplet, chemotherapy administered on an intent-to-treat basis. The assessment of the response to therapy for lung cancer mainly relied on the Response Evaluation Criteria in Solid Tumors (17). Paraffin-embedded pre-treatment tumor tissues were required to be available for immunohistochemistry. However, patients with a known history of cancer or without subsequent clinical follow-up data were excluded from the present study. Detailed clinicopathological and survival data, such as the tumor location, stage, differentiation, treatment, radiography review and outcome, were collected retrospectively. The current study was approved by the Institutional Review Board of The First Affiliated Hospital of Xi'an Jiaotong University. Each patient provided informed consent for participation in the present study.

Chemotherapy regimen. The chemotherapy regimens included the administration of $75-80 \mathrm{mg} / \mathrm{m}^{2}$ cisplatin or carboplatin, at a dose that was calculated to produce an area under the serum concentration-time curve of $6.0 \mathrm{~min} / \mathrm{mg} / \mathrm{ml}$, and a non-platinum agent. The treatment was repeated every 3 weeks. The same chemotherapy regimen was administered once every 3 weeks and drug doses were tailored according to patient tolerance, as necessary.

Assessment of treatment efficacy. The patients were monitored closely for respiratory and system symptoms, signs and disease relapse. Auxiliary examination was conducted using computed tomography, head magnetic resonance imaging and bone scanning every 3 months during treatment until the completion of chemotherapy, and every 3-6 months subsequently, until disease progression or the initiation of subsequent anticancer therapy was determined. The sum of the maximal diameters of all measurable tumor lesions was recorded at baseline and following treatment. These measurements were used to calculate the largest percentage reduction or smallest percentage increase in size using the baseline assessment as a reference. The best response to therapy was categorized using Response Evaluation Criteria In Solid Tumors 1.0 criteria (17). The pre- and post-treatment quality of life was also assessed.

Immunohistochemistry. Immunohistochemistry was performed to analyze the expression of the hCtr1, ATP7A and ATP7B proteins in the pre-treatment tumor tissue specimens. Therefore, $4 \mu \mathrm{m}$ thick tissue sections were prepared from a representative tumor tissue paraffin block for each patient. For immunohistochemical analysis, these tissue sections were deparaffinized and rehydrated, and then subjected to heat-induced antigen retrieval in citrate buffer in an autoclave oven. Subsequently, the sections were blocked for endogenous peroxidase activity in $3 \% \mathrm{H}_{2} \mathrm{O}_{2}$ /phosphate buffered saline (PBS) solution for $30 \mathrm{~min}$ at room temperature. The sections were then incubated overnight at $4^{\circ} \mathrm{C}$ with the primary antibody against hCtr1 (dilution, 1:250; Santa Cruz Biotechnology, Inc., Dallas, TX, USA), ATP7A (dilution, 1:100; Abcam, Cambridge, UK) or ATP7B (dilution, 1:100; Abcam). On the next day, the sections were washed with PBS three times, and then incubated with a biotin-labeled secondary antibody and the adenosine triphosphate binding cassette complex (LSAB kit; DAKO, Carpinteria, CA, USA) at $37^{\circ} \mathrm{C}$ for $20 \mathrm{~min}$.

For the color reaction, the sections were incubated with diaminobenzidine substrate solution as the chromogen. Finally, the sections were briefly counterstained with hematoxylin. Positive and negative controls were performed for all experiments. Ten fields of view per stained section (magnification, $\mathrm{x} 200$ ) were reviewed and scored under light microscopy (BX43; Olympus Corp, Tokyo, Japan) by two independent pathologists that were blinded to the clinical characteristics and outcomes of the patients. Each tissue section was semi-quantitatively scored on a 1-4 scale by estimating the percentage of positive cytoplasmic or membranous staining of tumor cells, as follows: 1, 1-25\% stained cells; 2, 26-50\% stained cells; $3,51-75 \%$ stained cells; and $4,>75 \%$ stained cells. Weakly positive cytoplasmic staining, with an intensity score of 1, was observed in certain pneumocytes and bronchiolar epithelial cells adjacent to tumor cells. With respect to the intensity and frequency, a high expression level was defined as tumor cells with diffuse cytoplasmic or membrane staining of moderate/strong intensity $(\geq 2)$, according to previous studies $(18,19)$.

Statistical analysis. The association between clinical characteristics and immunostaining were evaluated using $\chi^{2}$ or Fisher's exact tests. The overall survival (OS) time was calculated as the time interval between the date of pathological diagnosis and the date of mortality or last follow-up. The Kaplan-Meier method was used to generate survival curves for the OS time and the log-rank test was performed to evaluate the differences between the groups. Univariate logistic regression 


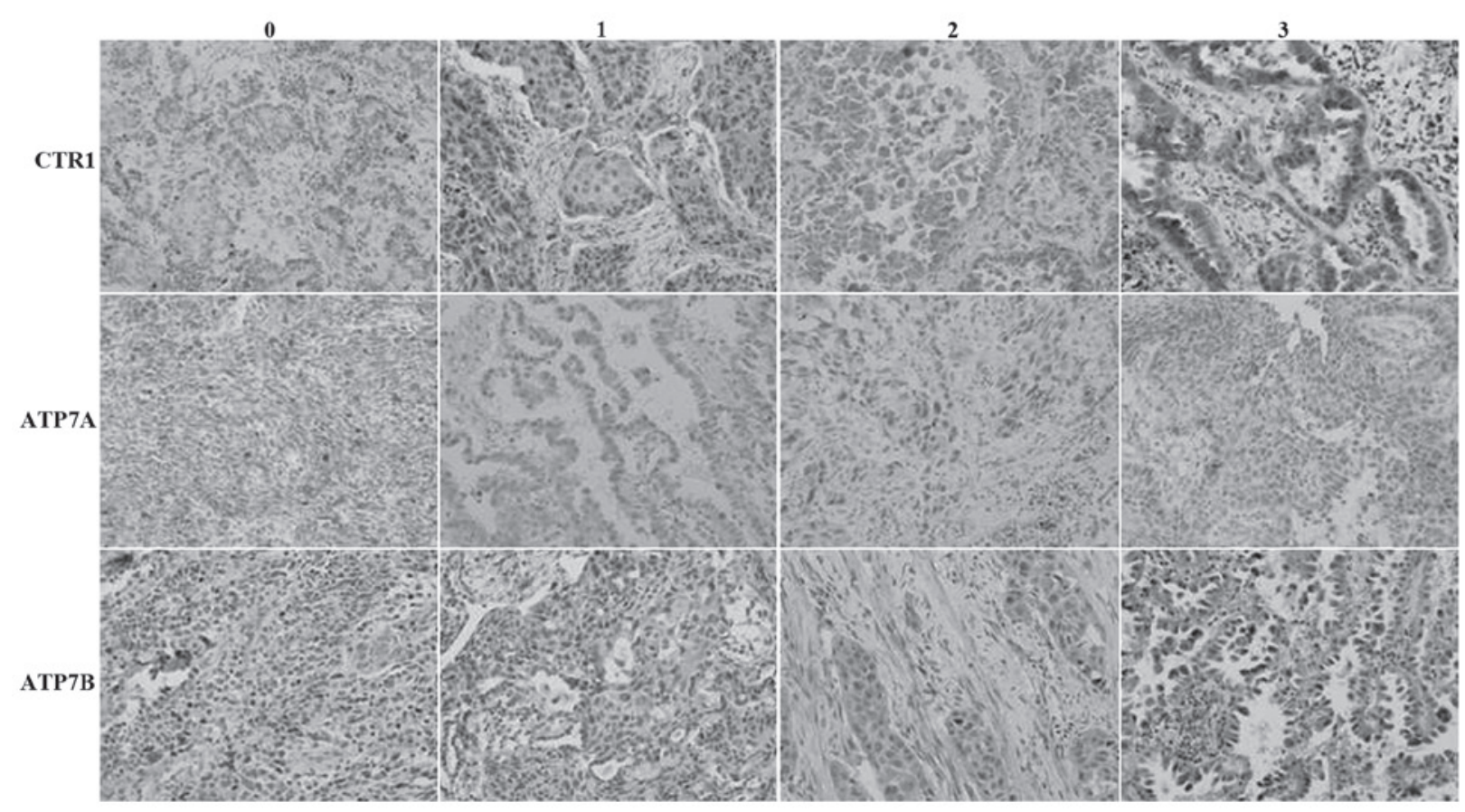

Figure 1. Immunohistochemical analysis of the expression of the (A) hCtrl, (B) ATP7A and (C) ATP7B proteins in non-small cell lung cancer tissue specimens. Magnification, x200. 0, no staining; 1, weak staining; 2, moderate staining; and 3, strong staining. CTR1, copper transporter 1; hCtr1, human CTR1; ATP7A, copper-transporting p-type adenosine triphosphatase 1; ATP7B, copper-transporting p-type adenosine triphosphatase 2.

analysis followed by multiple logistic regression analyses was applied to evaluate the association between protein expression and clinicopathological parameters as predictors of the treatment response. The association between potential predictors and survival time were evaluated by Cox proportional hazards models, using univariate and multivariate analyses. All statistical analyses were performed using Stata/SE 12.0 (StataCorp LP, College Station, TX, USA). $\mathrm{P}<0.05$ was considered to indicate a statistically significant difference.

\section{Results}

Patient characteristics and treatment responses. The present cohort of patients with NSCLC received platinum-based chemotherapy subsequent to the surgical removal of tumor lesions. The longest follow-up time was 66 months. The characteristics of patients are listed in Table I. In total, 14 patients were treated with gemcitabine and cisplatin (26\%), 12 with docetaxel and cisplatin (22\%), 15 (28\%) with vinorelbine and cisplatin, 7 (13\%) with paclitaxel and cisplatin, $5(9 \%)$ with pemetrexed and cisplatin, and $1(2 \%)$ with etoposide and cisplatin. The courses of chemotherapy ranged between 2 and 4 cycles. Stable disease was demonstrated by 4 patients, progressive disease was demonstrated by 15 patients and complete response was demonstrated by 1 patient.

Expression of the hCtrl, ATP7A and ATP $7 B$ proteins in NSCLC tissue specimens and association with measurable tumor response. The expression of the hCtr1, ATP7A and ATP7B proteins was observed in 59, 37 and 28\% of NSCLC tissue specimens, respectively (Fig. 1; Table I). The expression of ATP7B was significantly associated with tumor cell differentiation, while the expression of hCtr1 was significantly associated with improved chemotherapeutic responses. In total, 18 out of 33 patients with partial responses to chemotherapy demonstrated overexpression of the hCtr1 protein, whereas 14 out of 15 patients with progressive disease subsequent to chemotherapy demonstrated overexpression of the hCtr1 protein $(\mathrm{P}<0.05)$. However, the expression of the ATP7A and ATP7B proteins was not significantly associated with clinicopathological data. The expression of these copper transporters was not associated with patient age, histological tumor type, clinical stage or best measurable response (Table I).

Association between $h C t r l, A T P 7 A$ and ATP7B expression and patient survival. The median survival time was found to be 45 months in patients with low ATP7B expression in tumors, whereas it was 20 months in patients with high ATP7B expression in tumors, although the data were not statistically significant $(\mathrm{P}=0.162)$. Similarly, the median survival time was 20 months in patients with high tumor ATP7A expression, but $>66$ months in patients with low tumor ATP7A expression at the time of follow-up. The data were statistically significant $(\mathrm{P}<0.001)$.

Furthermore, the median survival time was 15 months in patients with low tumor hCtr1 expression, but $>66$ months in patients with high tumor hCtrl expression at the time of follow-up. This difference was statistically significant $(\mathrm{P}<0.001)$. High hCtr1 and low ATP7A expression were each favorable prognostic factors subsequent to chemotherapy in patients with resected NSCLC (Fig. 2).

In addition, the multivariate analysis data revealed that high hCtr1 expression combined with low ATP7A expression, tumor differentiation and patient gender were all favorable 


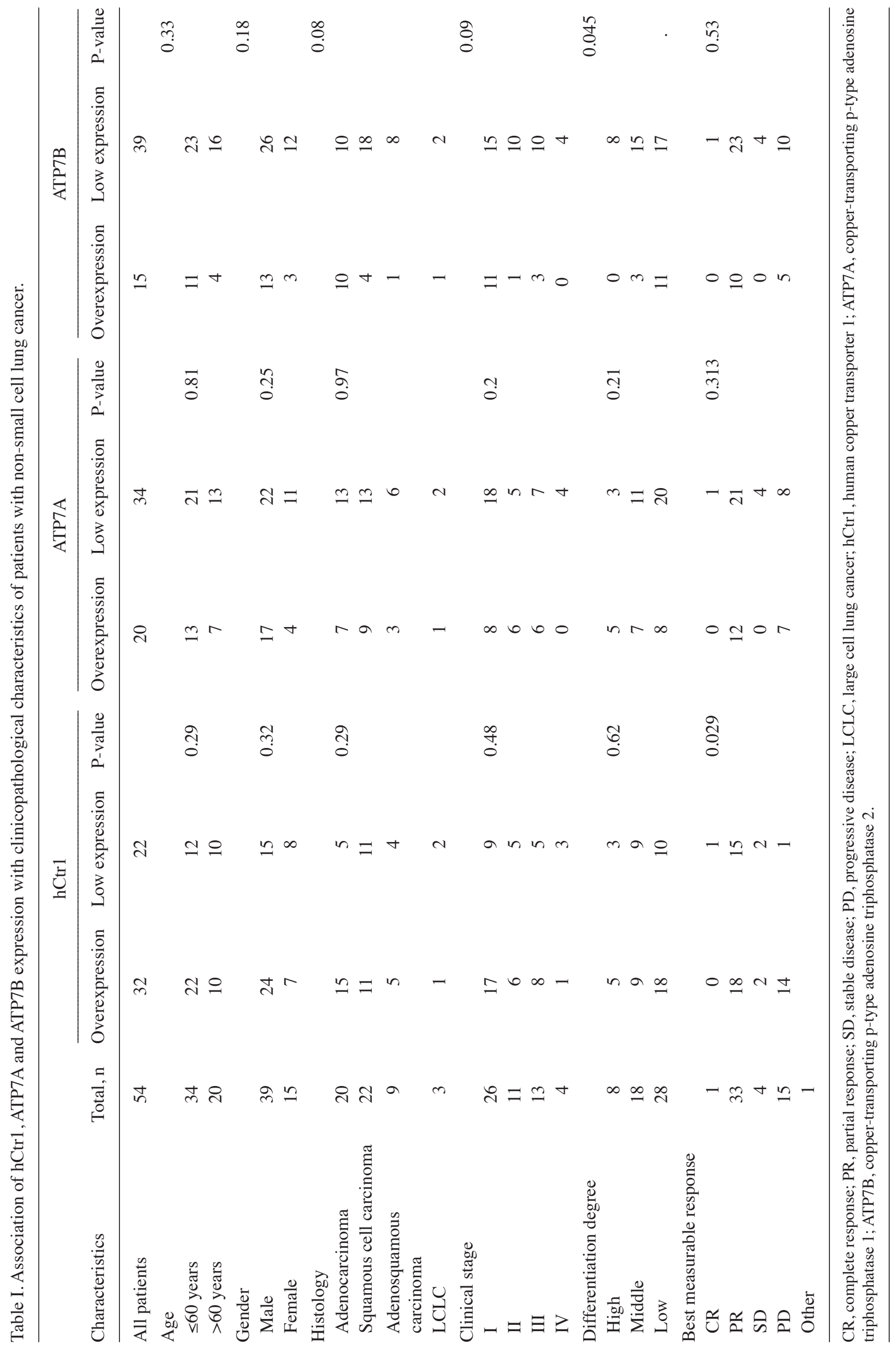


Table II. Univariate and multivariate analyses of prognostic factors for non-small cell lung cancer patients subsequent to treatment with platinum-based chemotherapy.

\begin{tabular}{|c|c|c|c|c|}
\hline \multirow[b]{2}{*}{ Variable } & \multicolumn{2}{|c|}{ Univariate analysis } & \multicolumn{2}{|c|}{ Multivariate analysis } \\
\hline & HR (95\% CI) & P-value & HR (95\% CI) & P-value \\
\hline \multicolumn{5}{|l|}{ CTR1 expression } \\
\hline High vs. low & $0.15(0.62-0.38)$ & 0.000 & $0.29(0.11-0.75)$ & 0.010 \\
\hline \multicolumn{5}{|l|}{ ATP7A expression } \\
\hline High vs. low & $7.20(2.48-20.87)$ & 0.000 & $3.68(1.11-12.12)$ & 0.032 \\
\hline \multicolumn{5}{|l|}{ ATP7B expression } \\
\hline High vs. low & $1.78(0.78-4.03)$ & 0.168 & $0.77(0.29-2.00)$ & 0.592 \\
\hline \multicolumn{5}{|l|}{ Gender } \\
\hline Male vs. female & $0.29(0.10-0.85)$ & 0.023 & $0.28(0.09-0.95)$ & 0.040 \\
\hline \multicolumn{5}{|l|}{ Age } \\
\hline$\geq 60$ vs. $<60$ years & $0.97(0.93-1.00)$ & 0.083 & $0.96(0.92-1.01)$ & 0.120 \\
\hline \multicolumn{5}{|l|}{ Tumor differentiation } \\
\hline Good/moderate vs. poor & $0.31(0.19-0.49)$ & 0.000 & $0.44(0.24-0.79)$ & 0.006 \\
\hline
\end{tabular}

HR, hazard ratio; CI, confidence interval; CTR1, copper transporter 1; ATP7A, copper-transporting p-type adenosine triphosphatase 1; ATP7B, copper-transporting p-type adenosine triphosphatase 2 .

A

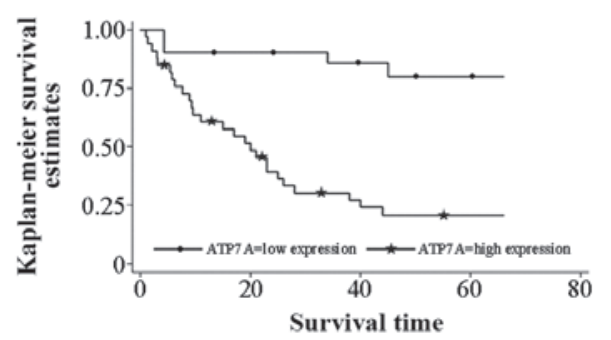

B

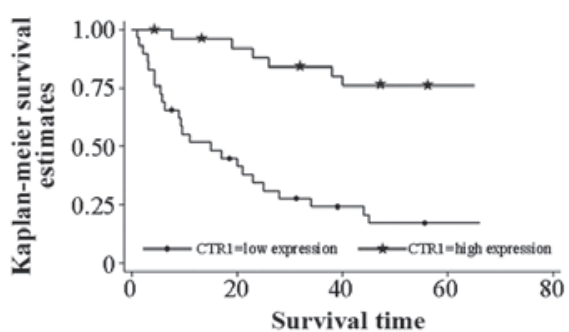

C

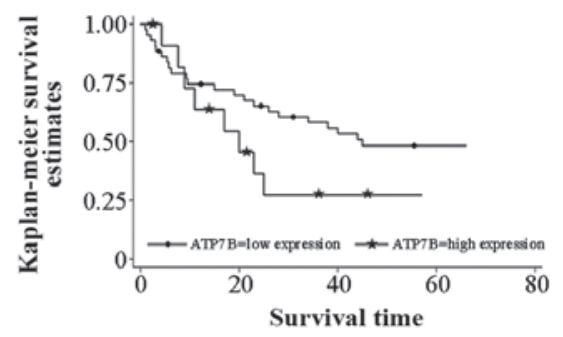

Figure 2. Kaplan-Meier survival curves stratified by the expression of the (A) human CTR1, (B) ATP7A, and (C) ATP7B proteins in non-small cell lung cancer tissue specimens. CTR1, copper transporter 1; ATP7A, copper-transporting p-type adenosine triphosphatase 1; ATP7B, copper-transporting p-type adenosine triphosphatase 2 .

independent predictive and prognostic factors for patients with resected NSCLC subsequent to chemotherapy (Table II).

\section{Discussion}

In the present study, the expression of the hCtr1, ATP7A and ATP7B proteins was analyzed in pre-treated NSCLC tissue specimens to determine the association between the expression of these proteins and chemosensitivity of the patients to platinum-based treatment. Out of the 54 lung cancer patients that underwent surgical tumor resection and first-line platinum-based doublet chemotherapy, significant overexpression of hCtr1, ATP7A and ATP7B was identified in 32 (59\%), 20 (37\%) and 15 (28\%) patients, respectively. In addition, ATP7B expression was significantly associated with tumor differentiation, and hCtrl expression was significantly associated with an improved chemotherapeutic response. The multivariate analysis revealed that high hCtrl expression, low ATP7A expression, tumor differentiation and patient gender were all favorable independent predictive and prognostic factors for patients with resected NSCLC that received chemotherapy. These data indicate that the detection of hCtr1 and ATP7A expression may be evaluated as biomarkers to predict the sensitivity of patients with NSCLC to platinum-based chemotherapy. High hCtr1 and low ATP7A expression were each favorable prognostic factors for patients with resected NSCLC subsequent to chemotherapy.

The present results revealed that the median survival time of patients subsequent to surgery combined with chemotherapy was longer and was markedly increased compared with the median survival time reported in the literature, which reports a median survival time of 9-11 months for lung cancer patients $(6,8)$. The cause of this discrepancy has not been elucidated and may be associated with the stage of the disease, radical resection of tumor lesions, duration of chemotherapy administration, the presence of metastatic tumors outside the lung and individual differences.

Platinum-based chemotherapy is the first-line chemotherapeutic treatment either prior to or following surgery $(20,21)$. However, drug resistance may be an issue and limit the efficacy of platinum-based chemotherapy in the clinic (22) and in the pre- and post-operative treatment of lung cancer (9). 
In general, copper is an essential element for cells, but excessive copper may be toxic or even lethal to cells. Therefore, mammalian cells have developed sophisticated mechanisms to maintain copper levels through intake, export and intracellular compartmentalization or buffering of copper. Ctr1 controls copper uptake, while ATP7A and ATP7B control the copper export from cells (23-25). Previous studies have identified that copper ion transport proteins are closely associated with the development of cisplatin resistance $(16,26,27)$. These copper transporters include Ctr1 and ATP7B and ATP7A $(23,28)$, which together regulate the overall intracellular cisplatin levels. It has been reported that the intracellular cisplatin level is an important determinant for the cytotoxic activity of cisplatin. Thus, detection of the expression of hCtr1, ATP7B and ATP7A in tumor lesions may provide an important insight into drug resistance in cisplatin-based cancer chemotherapy. In the present study, NSCLC tissue specimens were revealed to express various levels of hCtrl, ATP7A and ATP7B proteins and that ATP7B expression was significantly associated with tumor cell differentiation, while hCtrl expression was significantly associated with an improved chemotherapeutic response. Mechanistically, it is evident that hCtr1 expression upregulates drug levels in cells and therefore increases drug efficacy.

In addition, the present study also demonstrated that differential expression of these copper transporters, such as high hCtrl expression and low ATP7B and ATP7A expression, also contributed to a favorable prognosis in patients with resected NSCLC that received chemotherapy. Li et al (29) demonstrated that ATP7A expression was associated with the histological grade of NSCLC and the response to chemotherapy. Inoue et al (30) revealed that ATP7A mRNA expression was useful as a marker for cisplatin chemoresistance in NSCLC. Furthermore, Nakagawa et al (19) found that the ATP7B mRNA and protein levels were significantly increased in human NSCLC cisplatin-resistant xenografts compared with the levels in the cisplatin-sensitive xenografts, indicating that ATP7B was a useful biomarker for platinum resistance in NSCLC. However, Mangala et al (31) revealed that ATP7B was overexpressed in chemotherapy-sensitive ovarian carcinoma cells. However, the association between ATP7B overexpression and an unfavorable clinical outcome in various cancers has been reported (32). Overall, the present and published data indicate that the role of ATP7A and ATP7B in clinical cisplatin resistance may be complex and depend on factors including the patient population, tumor types, drug resistance mechanisms, treatment modalities and methods of data analysis (5). Thus, additional studies are required to clarify these issues.

The present study also revealed an association between $\mathrm{hCtr1}$ expression and curative effect, which is consistent with the findings of Choi et al (5). However, based on the cohort of patients in the present study, hCtrl expression may have prognostic value for predicting the treatment efficacy of chemotherapy using a cisplatin-based regimen combined with surgery. Ishida et al has previously reported that the expression of hCtr1 is associated with the clinical response in ovarian cancer $(33,34)$. Ogane et al $(35)$ revealed that CTR1 expression is a prognostic factor for outcome in endometrial cancer. There are numerous factors that contribute to cisplatin resistance, and additional studies are required to elucidate the molecular mechanism involved in the development of resistance to platinum-based chemotherapy in lung cancer patients. Cumulatively, the present results revealed that the detection of hCtr1 and ATP7A expression may be evaluated as a biomarker for the prediction of chemosensitivity to platinum-based chemotherapy; high hCtr1 and low ATP7A expression were each favorable prognostic factors for patients with resected NSCLC subsequent to chemotherapy. Surgery combined with neoadjuvant chemotherapy may improve the survival of NSCLC patients.

\section{Acknowledgements}

This study was supported by Shaanxi Science and Technology Research Funds (grant no., 2011K12-15).

\section{References}

1. Hildebrandt MA, Gu J and Wu X: Pharmacogenomics of platinum-based chemotherapy in NSCLC. Expert Opin Drug Metab Toxicol 5: 745-755, 2009.

2. Siegel R, Naishadham D and Jemal A: Cancer statistics, 2013. CA Cancer J Clin 63: 11-30, 2013.

3. Zornosa C, Vandergrift JL, Kalemkerian GP, Ettinger DS, Rabin MS, Reid M, Otterson GA, Koczywas M, D'Amico TA, Niland JC, et al: First-line systemic therapy practice patterns and concordance with NCCN guidelines for patients diagnosed with metastatic NSCLC treated at NCCN institutions. J Natl Compr Canc Netw 10: 847-856, 2012.

4. Ivy KD and Kaplan JH: A re-evaluation of the role of hcTR1, the human high-affinity copper transporter, in platinum-drug entry into human cells. Mol Pharmacol 83: 1237-1246, 2013.

5. Choi MK and Kim DD: Platinum transporters and drug resistance. Arch Pharm Res 29: 1067-1073, 2006.

6. Fisher MD and D'Orazio A: Phase II and III trials: Comparison of four chemotherapy regimens in advanced non small-cell lung cancer (ECOG 1594). Clin Lung Cancer 2: 21-22, 2000.

7. Schiller JH, Harrington D, Belani CP, et al: Comparison of four chemotherapy regimens for advanced non-small-cell lung cancer. N Engl J Med 346: 92-98, 2002.

8. Fossella F, Pereira JR, von Pawel J, et al: Randomized, multinational, phase III study of docetaxel plus platinum combinations versus vinorelbine plus cisplatin for advanced non-small-cell lung cancer: The tax 326 study group. J Clin Oncol 21: 3016-3024, 2003.

9. Liu JJ, Lu J and McKeage MJ: Membrane transporters as determinants of the pharmacology of platinum anticancer drugs. Curr Cancer Drug Targets 12: 962-986, 2012.

10. Mellor HR and Callaghan R: Resistance to chemotherapy in cancer: A complex and integrated cellular response. Pharmacology 81: 275-300, 2008.

11. Zisowsky J, Koegel S, Leyers S, et al: Relevance of drug uptake and efflux for cisplatin sensitivity of tumor cells. Biochem Pharmacol 73: 298-307, 2007.

12. Gupta A and Lutsenko S: Human copper transporters: Mechanism, role in human diseases and therapeutic potential. Future Med Chem 1: 1125-1142, 2009.

13. Kuo MT, Chen HH, Song IS, Savaraj N and Ishikawa T: The roles of copper transporters in cisplatin resistance. Cancer Metastasis Rev 26: 71-83, 2007.

14. Steiger D, Fetchko M, Vardanyan A, Atanesyan L, Steiner K, Turski ML, Thiele DJ, Georgiev O and Schaffner W: The drosophila copper transporter ctrlC functions in male fertility. J Biol Chem 285: 17089-17097, 2010.

15. Liang ZD, Long Y, Tsai WB, et al: Mechanistic basis for overcoming platinum resistance using copper chelating agents. Mol Cancer Ther 11: 2483-2494, 2012.

16. Chen HH, Yan JJ, Chen WC, et al: Predictive and prognostic value of human copper transporter 1 (hctr1) in patients with stage III non-small-cell lung cancer receiving first-line platinum-based doublet chemotherapy. Lung Cancer 75: 228-234, 2012.

17. Taylor PT and Haverstick D: Re: New guidelines to evaluate the response to treatment in solid tumors (ovarian cancer). J Natl Cancer Inst 97: 151, 2005. 
18. Holzer AK, Varki NM,Le QT, GibsonMA Naredi P and Howell SB: Expression of the human copper influx transporter 1 in normal and malignant human tissues. J Histochem Cytochem 54: 1041-1049, 2006.

19. Nakagawa T, Inoue Y, Kodama H, Yamazaki H, Kawai K, Suemizu H, Masuda R, Iwazaki M, Yamada S, Ueyama Y, et al: Expression of copper-transporting p-type adenosine triphosphatase (ATP7B) correlates with cisplatin resistance in human non-small cell lung cancer xenografts. Oncol Rep 20: 265-270, 2008.

20. Konstantakou EG, Voutsinas GE, Karkoulis PK, Aravantinos G, Margaritis LH and Stravopodis DJ: Human bladder cancer cells undergo cisplatin-induced apoptosis that is associated with p53-dependent and p53-independent responses. Int J Oncol 5: 401-416, 2009.

21. Köberle B, Tomicic MT, Usanova S and Kaina B: Cisplatin resistance: Preclinical findings and clinical implications. Biochim Biophys Acta 1806: 172-182, 2010.

22. Abada P and Howell SB: Regulation of cisplatin cytotoxicity by $\mathrm{cu}$ influx transporters. Metal Based Drugs 2010: 317581, 2010.

23. Lenartowicz M and Krzeptowski W: Structure and function of ATP7A and ATP7B proteins-Cu-transporting ATPases. Postepy Biochem 56: 317-327, 2010.

24. Zhou B and Gitschier J: Hctr1: A human gene for copper uptake identified by complementation in yeast. Proc Natl Acad Sci USA 94: 7481-7486, 1997.

25. Cox DW and Moore SD: Copper transporting p-type atpases and human disease. J Bioenerg Biomembr 34: 333-338, 2002.

26. Howell SB, Safaei R, Larson CA and Sailor MJ: Copper transporters and the cellular pharmacology of the platinum-containing cancer drugs. Mol Pharmacol 77: 887-894, 2010.

27. Zhang Y, Li M, Yao Q and Chen C: Roles and mechanisms of copper transporting atpases in cancer pathogenesis. Med Sci Monit 15: RA1-RA5, 2009.

28. Furukawa T, Komatsu M, Ikeda R, Tsujikawa K and Akiyama S: Copper transport systems are involved in multidrug resistance and drug transport. Curr Med Chem 15: 3268-3278, 2008.
29. Li ZH, Qiu MZ, Zeng ZL, Luo HY, Wu WJ, Wang F, Wang ZQ, Zhang DS, Li YH and Xu RH: Copper-transporting p-type adenosine triphosphatase (ATP7A) is associated with platinum-resistance in non-small cell lung cancer (NSCLC). J Transl Med 10: 21, 2012.

30. Inoue Y, Matsumoto H, Yamada S, Kawai K, Suemizu H, Gika M, Takanami I, Iwazaki M and Nakamura M: Association of ATP7A expression and in vitro sensitivity to cisplatin in non-small cell lung cancer. Oncol Lett 1: 837-840, 2010.

31. Mangala LS, Zuzel V, Schmandt R, Leshane ES, Halder JB, Armaiz-Pena GN, Spannuth WA, Tanaka T, Shahzad MM, Lin YG, et al: Therapeutic targeting of ATP7B in ovarian carcinoma. Clin Cancer Res 15: 3770-3780, 2009.

32. Nakayama K, Kanzaki A, Terada K, Mutoh M, Ogawa K, Sugiyama T, Takenoshita S, Itoh K, Yaegashi N, Miyazaki K, et al: Prognostic value of the $\mathrm{Cu}$-transporting ATPase in ovarian carcinoma patients receiving cisplatin-based chemotherapy. Clin Cancer Res 10: 2804-2811, 2004.

33. Yoshida H, Teramae M, Yamauchi M, Fukuda T, Yasui T, Sumi T, Honda K and Ishiko O: Association of copper transporter expression with platinum resistance in epithelial ovarian cancer. Anticancer Res 33: 1409-1414, 2013.

34. Ishida S, McCormick F, Smith-McCune K and Hanahan D: Enhancing tumor-specific uptake of the anticancer drug cisplatin with a copper chelator. Cancer Cell 17: 574-583, 2010.

35. Ogane N, Yasuda M, Kameda Y, Yokose T, Kato H, Itoh A, Nishino S, Hashimoto Y and Kamoshida S: Prognostic value of organic anion transporting polypeptide 1B3 and copper transporter 1 expression in endometrial cancer patients treated with paclitaxel and carboplatin. Biomed Res 34: 143-151, 2013. 